\title{
DNA Methylation Markers for Pan-Cancer Prediction by Deep Learning
}

\author{
Biao Liu ${ }^{1,+}{ }^{\mathbb{C}}$, Yulu Liu ${ }^{1,+}$, Xingxin Pan ${ }^{1} \mathbb{D}$, Mengyao Li ${ }^{2}$, Shuang Yang ${ }^{1, *}$ and Shuai Cheng $\mathrm{Li}^{3, *}$ \\ 1 BGI Education Center, University of Chinese Academy of Sciences, Shenzhen 518083, China; \\ biaoliu2019@gmail.com (B.L.); liuyulu@genomics.cn (Y.L.); panxingxin16@mails.ucas.ac.cn (X.P.) \\ 2 Research and Development Department, Shenzhen Byoryn Technology Co.,Ltd, Shenzhen 518000, China; \\ limengyao007@gmail.com \\ 3 Department of Computer Science, City University of Hong Kong, Kowloon 999077, Hong Kong, China \\ * Correspondence: yangsh@genomics.cn (S.Y.); shuaicli@gmail.com (S.C.L.) \\ + These authors contributed equally to this work.
}

Received: 27 June 2019; Accepted: 30 September 2019; Published: 4 October 2019

check for updates

\begin{abstract}
For cancer diagnosis, many DNA methylation markers have been identified. However, few studies have tried to identify DNA methylation markers to diagnose diverse cancer types simultaneously, i.e., pan-cancers. In this study, we tried to identify DNA methylation markers to differentiate cancer samples from the respective normal samples in pan-cancers. We collected whole genome methylation data of 27 cancer types containing 10,140 cancer samples and 3386 normal samples, and divided all samples into five data sets, including one training data set, one validation data set and three test data sets. We applied machine learning to identify DNA methylation markers, and specifically, we constructed diagnostic prediction models by deep learning. We identified two categories of markers: $12 \mathrm{CpG}$ markers and 13 promoter markers. Three of $12 \mathrm{CpG}$ markers and four of 13 promoter markers locate at cancer-related genes. With the CpG markers, our model achieved an average sensitivity and specificity on test data sets as $92.8 \%$ and $90.1 \%$, respectively. For promoter markers, the average sensitivity and specificity on test data sets were $89.8 \%$ and $81.1 \%$, respectively. Furthermore, in cell-free DNA methylation data of 163 prostate cancer samples, the CpG markers achieved the sensitivity as $100 \%$, and the promoter markers achieved $92 \%$. For both marker types, the specificity of normal whole blood was 100\%. To conclude, we identified methylation markers to diagnose pan-cancers, which might be applied to liquid biopsy of cancers.
\end{abstract}

Keywords: biomarker; methylation; pan-cancer; deep learning; CpG; promoter

\section{Introduction}

DNA methylation, as an important epigenetic modification, is associated with gene silencing, and the primary methylated sequence in vertebrates is CpG [1,2]. CpG methylations located at promoter silence the promoter activity, thus, they are negative correlated with the gene expression $[3,4]$. Furthermore, promoter methylations play major roles in cancers by suppressing transcription of some vital genes, such as tumor suppressor genes $[5,6]$.

Since DNA methylation plays an important role in cancers, many studies have utilized DNA methylated sequences as biomarkers for cancer detections, including CpG markers and promoter markers. Specifically, irregular methylations in promoters of cancer-related genes could serve as biomarkers for early cancer diagnosis and prognosis [7]. For example, adenomatous polyposis coli (APC) promoter methylation could be a biomarker for early diagnosis of prostate cancer [8], and O6-methylguanine-DNA-methyltransferase (MGMT) promoter methylation might be a predictive 
biomarker for cancer prognosis [9]. For CpG markers, ten diagnosis markers and eight prognosis markers in circulating tumor DNA of hepatocellular carcinoma have been screened [10].

Although quite a few DNA methylation biomarkers have been identified, and some of them have even been commercialized [11], one of the common limits is that these markers can only apply to one or few cancer types. Studying the similarities and differences among diverse cancer types is known as pan-cancer analysis, which has revealed that some different cancer types could have similar methylation patterns, and biomarkers that cross boundaries among diverse cancer types are expected to be identified $[12,13]$. Although some pan-cancer differentially methylated CpG sites have been identified [14,15], effective and practical pan-cancer methylation biomarkers remain to be identified. In this study, we focused on identifying DNA methylation biomarkers, including CpG markers and promoter markers, for diagnosing pan-cancers. We collected the whole genome methylation data of 27 cancer types containing 10,140 cancer samples and 3386 normal samples from TCGA (The Cancer Genome Atlas) [12] and GEO (Gene Expression Omnibus) [16]. Then, we used machine learning to analyze and identify cancer-special CpG markers and promoter markers. Specifically, we constructed diagnostic prediction models by deep learning. Finally, we identified $12 \mathrm{CpG}$ markers and 13 promoter markers, which can be used to predict pan-cancer precisely.

\section{Materials and Methods}

\subsection{Datasets}

We totally collected whole genome methylation data of 10,140 cancer samples and 3386 normal samples from TCGA and GEO. Specifically, methylation data of 4840 cancer samples and 1742 matched normal samples (matched normal sample: healthy tissue adjacent to tumor from the same patient) were divided randomly into a training data set (named as Training data set), a validation data set (named as Validation data set), and a test data set (named as Test data set 1) (Table S1). To make markers more adaptive in virtual normal samples, we added 727 cancer samples and 836 normal samples (most of them were virtual normal samples) into the Training data set (virtual normal sample: healthy tissue from healthy, unrelated individuals). Therefore, the Training data set contained 4827 cancer samples and 2716 normal samples (Tables S2 and S3). Both Validation data set and Test data set 1 contained 370 cancer samples and 201 matched normal samples from eight cancer types (Tables S1 and S2); The other two test data sets are named as Test data set 2 and Test data set 3 . Test data set 2 contained 3041 cancer samples and 268 matched normal samples from 15 cancer types (Table S4); Test data set 3 contained 1532 cancer samples and 540 virtual normal samples from five cancer types (Table S5). The methylation data of each sample came from Illumina's Infinium HumanMethylation450 BeadChip, which contains more than 450,000 methylation sites. The details of all samples are summarized in Tables S2-S6. We calculated the average methylation beta value of all CpG sites located in the promoter of the same gene as methylation beta value of the promoter. Specifically, upstream $1500 \mathrm{bp}$ of TSS (Transcription start site) to downstream $500 \mathrm{bp}$ of TSS are defined as a promoter [17,18]. We removed $\mathrm{CpG}$ sites and promoters where at least one sample had missed value to guarantee more strict data sets. Finally, 139,422 of 485,000 CpG sites and 15,316 of 24,062 promoters were left for the following analysis. Therefore, all samples had 139,422 common CpG sites and 15,316 common promoters. Data containing CpG sites and data containing promoters were analyzed parallelly in the following steps.

\subsection{Identifying Markers}

For mining markers, first we used the 'moderated t-statistics' method [19] to conduct the prescreening procedure to get the methylation sites with the most differential methylation expression. This method utilized Empirical Bayes for shrinking the variance and Benjamini-Hochberg procedure [20] to adjust $p$ values. We sorted all candidate markers by the adjusted $p$ values from low to high (lower adjusted $p$ value means that the differential rates of methylation between cancer samples and normal samples are larger), and we took the top 2000 markers as the next candidate markers. 
Next, we used two strategies to obtain fewer markers. One machine learning strategy is LASSO (least absolute shrinkage and selection operator) [21] under a binomial distribution. We randomly subsampled 75 percent of the samples every time and conducted LASSO procedure to identify markers with the biggest methylation beta value difference. After 1000 times sampling, we selected the markers that were chosen by LASSO at least 750 times. In this process, we did not choose the minimum lambda but chose the "1-se" lambda which is one standard error larger than the minimum to make the model simpler. Besides, the minimization goal we chose was 'auc' to make our model more robust. 10-fold cross-validation was applied each time. Another machine learning strategy is a random forest. The tree number to use for the first forest was 5000 and for all additional forests was 2000. The algorithm we applied used OOB (Out-of-bag) error as minimization criterion, and removed those least important variables from the random forest [22]. At each iteration, we set the dropping fraction of variables at 0.3 . Four main R packages ('limma', 'glmnet', 'doParallel', and 'varSelRF') were implemented in R version 3.5.0 to conduct these three machine learning strategies.

\subsection{Constructing Diagnostic Prediction Models}

To construct diagnostic prediction models, we constructed two multi-layer feedforward neural networks, both of which contained one input layer, multiple hidden layers and one output layer. The source code we used for prediction is publicly available at https://github.com/BiaoLiu2017/Cancermethylation. The input layer was namely the input data matrix (data matrices only containing marker sites), and the output layer had just one neural unit, whose activation function was sigmoid activation function while the activation function of hidden units was ReLU. For each hidden layer, the number of hidden units was the same. The cost function of the neural network was standard logistic regression cost function. The optimization algorithm we deployed in the network was Adam optimization algorithm, and the exponential decay rate for the first moment estimates was 0.9 , while the second was 0.999 . The learning rate decay strategy was an exponential decay, which means the learning rate would multiple a decay rate after specified epochs. To prevent overfitting, we carried out a batch normalization after activation function of every hidden layer. Another strategy to prevent overfitting is early stopping, and we chose a befitting training point to stop to make the model more suitable for the Validation data set. We conducted a random search [23] for hyper-parameter optimization. Table S7 shows the hyper-parameters we tuned in the process of training the neural network. In other words, we adopted the strategy that randomly initializes these hyper-parameters in the range as Table S7. We parallelly trained 1000 neural networks, and finally chose the hyper-parameters combination that had the best performance for the Validation data set. The final hyper-parameters combination is the best scheme as Table S7 shows. We deployed the best hyper-parameters into the final deep learning models and trained them by feeding the Training data set. We used the Validation data set to justify whether the model was overfitting. After we trained two neural network models whose performance were good enough in the Validation data set, we tested our diagnostic prediction models in Test data set 1 . Furthermore, to evaluate the performance of our model unbiasedly, we tested our prediction models in the other two test data sets: Test data set 2 and Test data set 3 . What needs to be emphasized is that all three test data sets were tested just once. The reason we divided samples in this way was to evaluate whether our model could predict untrained cancers. Before being fed into deep learning models, all data were subjected to standardization to fit a standardized normal distribution; namely the average was 0 , and the standard deviation was 1 . The deep neural network models that we deployed were based on the deep learning framework Tensorflow-GPU version 1.4.0 [24]. Logistic regression required scikit-learn version 0.19.1. We obtained SHAP (SHapley Additive exPlanation) values [25] by executing package 'shap' to interpret model predictions. To evaluate the robustness of markers we selected, random sampling of 100 times were carried out. Each time, 6000 samples were random selected from all samples. Each data set was requested to have the same ratio of cancer and normal as that of the original data set. Additionally, $<30 \%$ sample overlap among all 100 data sets was required 
too. Each data set was divided into one training data set and one test data set with same ratio of cancer and normal.

\section{Results}

\subsection{Identifying Cancer-Specific Methylation Markers by Machine Learning}

We utilized the Training data set to analyze and identify methylation markers by three machine learning methods. Figure 1 shows the procedure of identifying methylation marker. We organized the Training data set into two data matrices: CpG methylation matrix and promoter matrix. The CpG methylation matrix consisted of beta values of 139,422 CpG methylation sites, and promoter matrix consisted of beta values of 15,316 promoters. These two data matrices were utilized to identity the CpG markers and the promoter markers. First, a prescreening procedure was conducted by 'moderated t-statistics', to identify candidate markers with the most differential methylation beta value between cancer samples and normal samples. After that, we obtained the top 2000 markers as the candidate markers, including $2000 \mathrm{CpG}$ markers and 2000 promoter markers. Next, we used two strategies to reduce the number of markers parallelly. One machine learning strategy was LASSO (least absolute shrinkage and selection operator) under a binomial distribution. After 1000 times sampling, we selected the markers that were chosen by LASSO at least 750 times. Eventually, by LASSO we got $63 \mathrm{CpG}$ markers and 68 promoter markers. Another machine learning strategy was random forest, and we got $115 \mathrm{CpG}$ markers and 57 promoter markers. We took 12 overlapping CpG markers (Table 1) and 13 overlapping promoter markers (Table 2) between these two machine learning methods as final markers. In $12 \mathrm{CpG}$ markers, reference genes of three markers involve cancer-related pathway. SOX14 (cg04374393 locates at the promoter of SOX14 gene) involves molecular mechanisms of cancer; TP73 (reference gene of cg17804348) involves p53 signaling pathway; SND1 (cg26642667 locates at the promoter of SND1 gene) involves viral carcinogenesis. In 13 promoter markers, associated genes of four markers involve cancer-related pathway. ACVRL1 involves TGF-beta signaling pathway; AURKB involves regulation of TP53 activity; RHOT2 involves mitophagy; WT1 involves transcriptional misregulation in cancer.

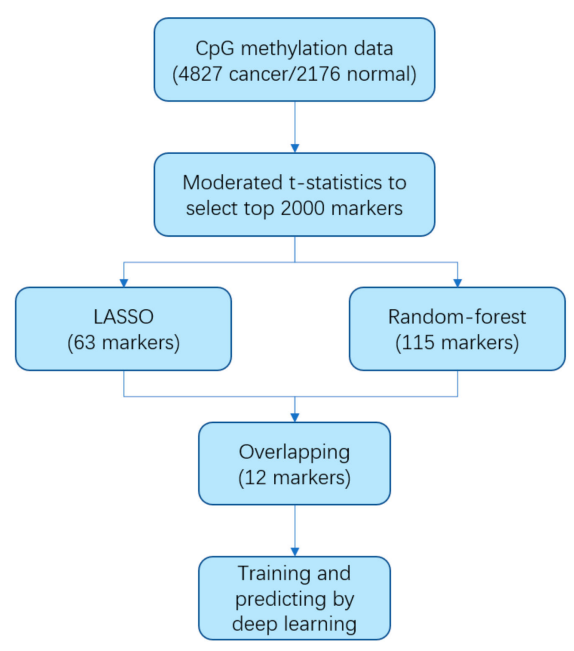

(a)

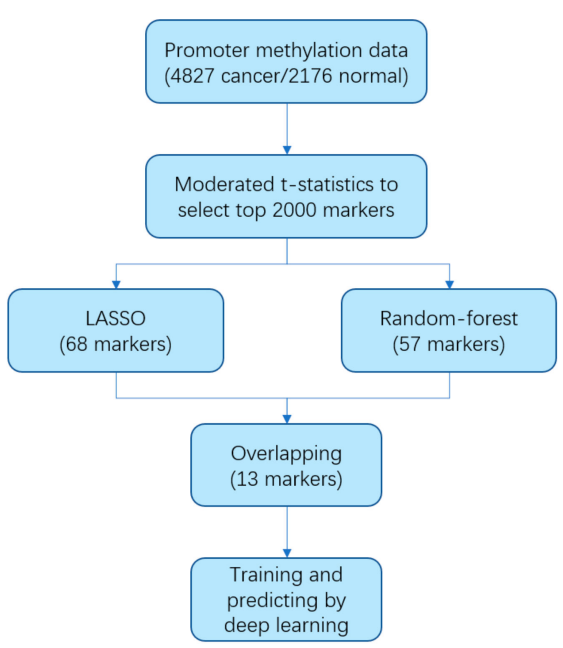

(b)

Figure 1. Workflow chart of identifying markers by machine learning. (a) Workflow of CpG methylation data. (b) Workflow of promoter methylation data. CpG methylation data contained 139,422 CpG sites, and promoter methylation data contained 15,316 promoters. We utilized the Training data set containing 4827 cancer samples and 2176 normal samples to identify markers applying three machine learning strategies (Moderated t-statistics, LASSO, and Random-forest) and obtained 12 markers for the CpG methylation data, and 13 markers for the promoter methylation data. Then, we trained two deep learning models for $\mathrm{CpG}$ markers and promoter markers respectively. 
Table 1. Characteristics of $12 \mathrm{CpG}$ markers in the Training data set.

\begin{tabular}{cccccc}
\hline Markers & Ref Gene & Coefficients & SE & $z$ Value & $p$ Value \\
\hline & & 4.28017 & 0.12365 & 34.614 & $<0.001$ \\
cg01397449 & EXOC3L1 & -1.26195 & 0.0828 & -15.241 & $<0.001$ \\
cg04374393 & SOX14 & 0.44095 & 0.10759 & 4.098 & $<0.001$ \\
cg06575035 & PCDHGA1 & 1.0089 & 0.09321 & 10.823 & $<0.001$ \\
cg07333191 & Chr4:13 & 0.5435 & 0.11389 & 4.772 & $<0.001$ \\
cg16389386 & Chr7:154 & -0.38554 & 0.06408 & -6.016 & $<0.001$ \\
cg16508627 & HS3ST2 & -0.54732 & 0.11407 & -4.798 & $<0.001$ \\
cg16926102 & Chr10:23 & 0.8946 & 0.11951 & 7.486 & $<0.001$ \\
cg17804348 & TP73 & 1.09724 & 0.06442 & 17.033 & $<0.001$ \\
cg19710323 & Chr12:34 & -0.8628 & 0.10259 & -8.41 & $<0.001$ \\
cg22620090 & Chr6:104 & 0.36339 & 0.07759 & 4.683 & $<0.001$ \\
cg26642667 & SND1 & -0.85746 & 0.04911 & -17.461 & $<0.001$ \\
cg26733975 & RP11-760D2.1 & -0.97163 & 0.10248 & -9.481 & $<0.001$ \\
\hline
\end{tabular}

Note: SE indicates standard errors of coefficients; $z$ value indicates Wald $z$-statistic value.

Table 2. Characteristics of 13 promoter markers in the Training data set.

\begin{tabular}{ccccc}
\hline Markers & Coefficients & SE & $z$ Value & $p$ Value \\
\hline & 2.6472 & 0.5316 & 4.979 & $<0.001$ \\
ACVRL1 & 5.5848 & 0.7523 & 7.423 & $<0.001$ \\
AURKB & -3.9969 & 1.2242 & -3.265 & 0.001 \\
GRASPOS & -1.0094 & 0.3599 & -2.805 & 0.005 \\
MC3R & -12.2853 & 0.858 & -14.319 & $<0.001$ \\
OR10H2 & -6.8254 & 0.6101 & -11.188 & $<0.001$ \\
OTX2-AS1 & 3.664 & 0.6136 & 5.972 & $<0.001$ \\
PCDHGA12 & 0.6188 & 0.5294 & 1.169 & 0.242 \\
PCDHGA5 & 1.8653 & 0.704 & 2.649 & 0.008 \\
PCDHGA6 & 1.0961 & 0.6552 & 1.673 & 0.094 \\
PHC3 & -12.865 & 0.9678 & -13.293 & $<0.001$ \\
RHOT2 & 11.3143 & 0.8959 & 12.628 & $<0.001$ \\
TOX2 & 3.039 & 0.8061 & 3.77 & $<0.001$ \\
WT1 & 4.5058 & 0.4796 & 9.394 & $<0.001$ \\
\hline
\end{tabular}

Note: SE indicates standard errors of coefficients; $z$ value indicates Wald $z$-statistic value.

\subsection{Constructing Diagnostic Prediction Models by Deep Learning}

The markers obtained by machine learning were used to classify and predict cancer and normal samples by deep learning method. We constructed two multi-layer feedforward neural networks based on the deep learning framework Tensorflow and fed the Training data set into these two deep neural network models. We utilized a random search for hyper-parameter optimization, and Table S7 shows the best hyper-parameter combination. These two deep learning models were deployed with the best hyper-parameters and trained again. Figure S1 shows the training curves. By early stopping strategy, we chose a befitting training point to stop, to make the model more suitable for the Validation data set. After obtaining the best parameters, we tested our deep learning models in the three test data sets (Test data set 1, Test data set 2, and Test data set 3). Figure 2 shows the ROC (Receiver operating characteristic) curves of both two marker types. AUC (Area under the Curve of ROC) of Test data set 1 is 0.989 for CpG markers, and 0.985 for promoter markers. Figure 3 shows the results of unsupervised hierarchical clustering for Training data set, Validation data set, and Test data set 1 , while Figure 4 shows the results for the other two test data sets (Test data set 2 and Test data set 3). These results indicate that cancer samples can be distinguished markedly from normal samples by both two marker types. Table 3 shows a summary of all prediction results for both CpG markers and promoter markers (More details see Tables 4-7). Figure 5 shows the distribution of predict values in all samples. For CpG markers, average sensitivity and specificity of three test data sets were $92.8 \%$ 
and $90.1 \%$ respectively (Table 3). For promoter markers, average sensitivity and specificity of three test data sets were $89.8 \%$ and $81.1 \%$ respectively (Table 3 ). Although sensitivity and specificity in most cancer types were higher than 0.7 for both two marker types, specificity of esophagus and stomach cancer for promoter markers were lower than 0.6 , and the sensitivity of oral, thyroid, and nasopharynx cancer for promoter markers were lower than 0.6 (Tables 4-7). Therefore, all 27 cancer types could be diagnosed precisely by $\mathrm{CpG}$ markers, while only twenty-two of 27 cancer types could be diagnosed precisely by promoter markers. Both two categories of markers predicted the same results in each of $88.4 \%$ samples (i.e., 5262 samples) of three test data sets (i.e., 5952 samples), and average sensitivity and specificity of these 5262 samples were promoted to $96.4 \%$ and $91.6 \%$. Therefore, if the prediction result of one sample is same between CpG markers and promoter markers, the prediction will be more reliable. Average sensitivity and specificity in Test data set 1 were much higher than Test data set 2 and Test data set 3 for both two categories of markers, which means the models we trained are more adapted to eight trained tissue types than the other 20 untrained tissue types. Furthermore, for CpG markers, sensitivity and specificity of the eight cancers (breast, kidney, liver, lung, bile duct, lymph nodes, cervix, and skin cancer) were both higher than 95\% (Tables 4-7). Additionally, for promoter markers, sensitivity and specificity of nine cancers (breast, colorectal, liver, lung, adrenal gland, bile duct, soft tissue, cervix, and skin cancer) were both higher than 95\% (Tables 4-7). Conclusively, the prediction results indicate that our deep learning models can correctly classify cancer samples and normal samples in pan-cancers.

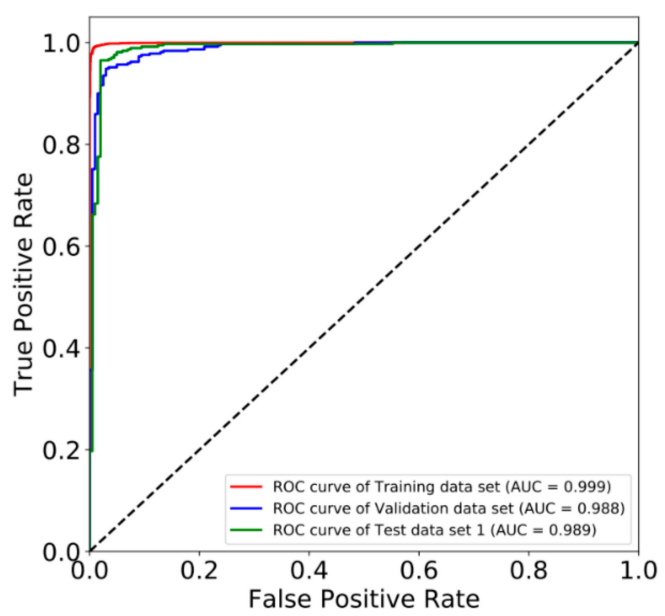

(a)

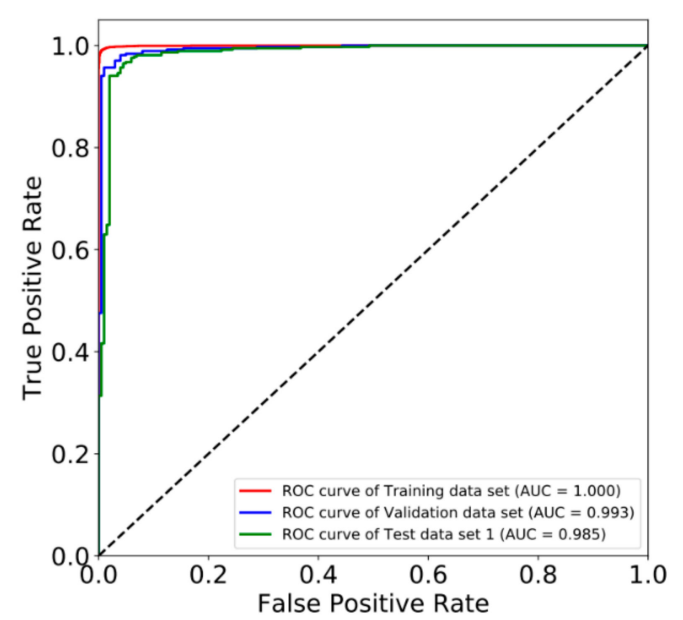

(b)

Figure 2. ROC curves of the three data sets. (a) ROC curves of CpG methylation data. (b) ROC curves of promoter methylation data. ROC curves: Receiver operating characteristic curves. 


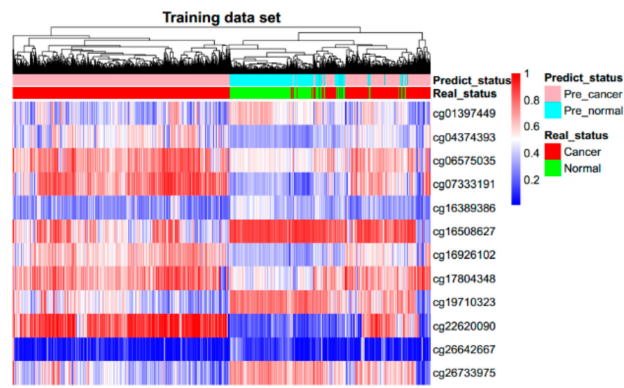

(a)

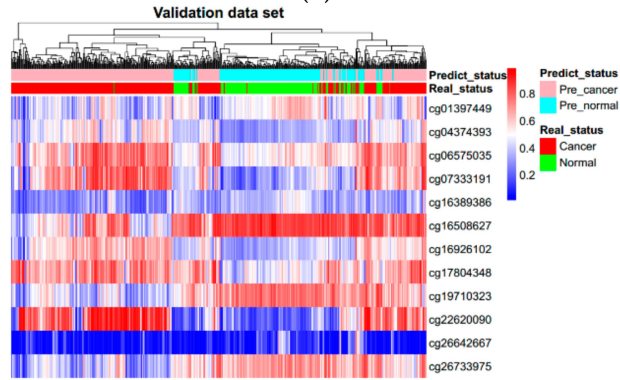

(c)

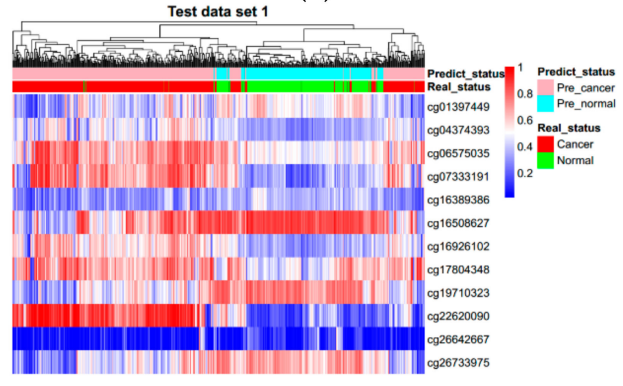

(e)

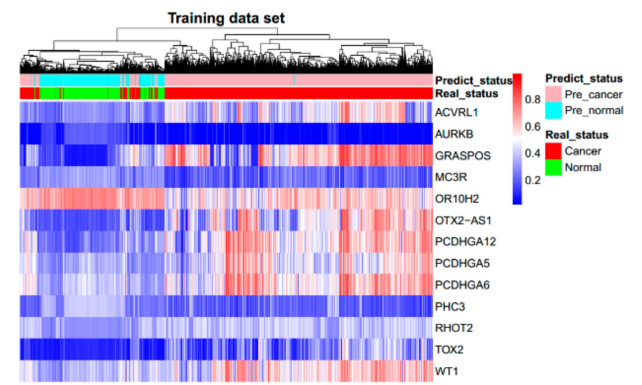

(b)

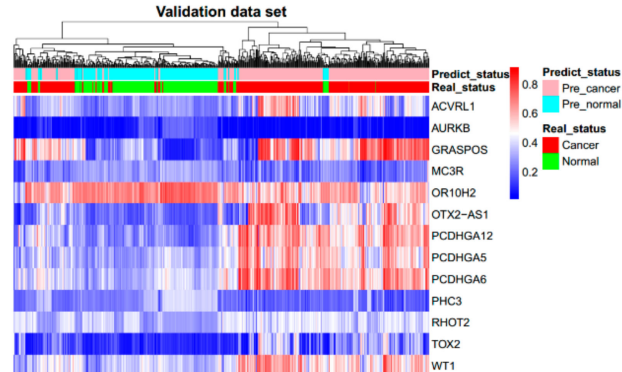

(d)

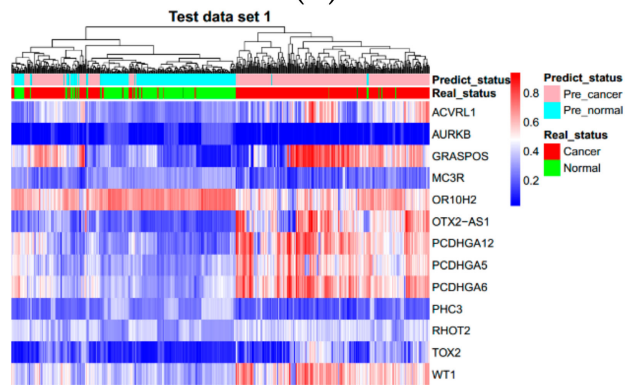

(f)

Figure 3. Unsupervised hierarchical clustering of the three data sets. (a,c,e) come from $12 \mathrm{CpG}$ markers and $(\mathbf{b}, \mathbf{d}, \mathbf{f})$ come from 13 promoter markers. Methylation beta values range from 0 to 1 .

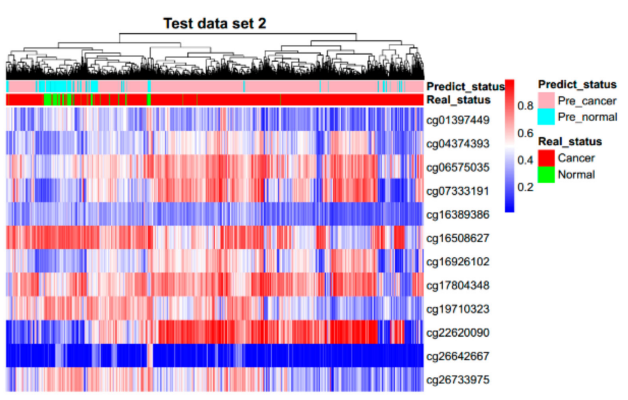

(a)

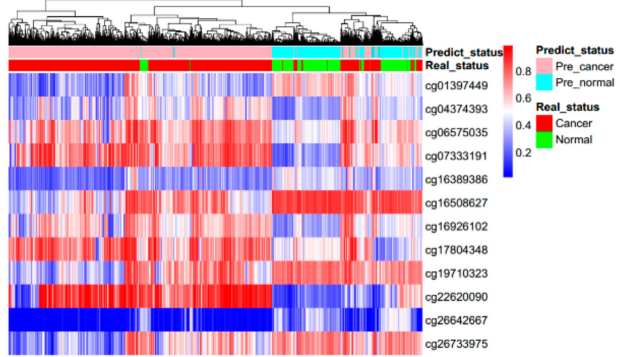

(c)

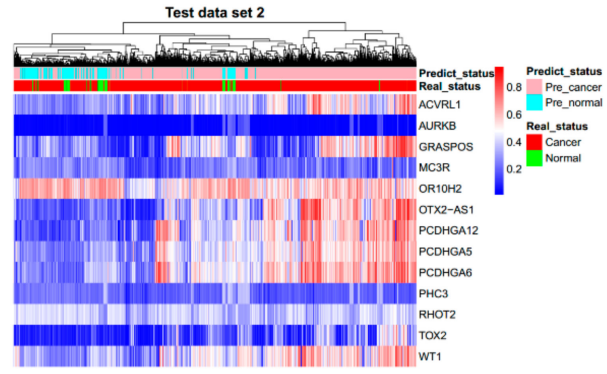

(b)

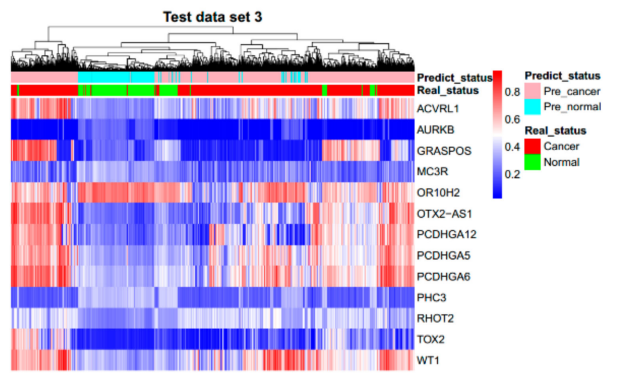

(d)

Figure 4. Unsupervised hierarchical clustering of Test data set 2 and Test data set 3 . (a,c) come from 12 CpG markers and (b,d) come from 13 promoter markers. Methylation beta values range from 0 to 1 . 
Table 3. The summary of all prediction results

\begin{tabular}{|c|c|c|c|c|c|c|c|c|c|c|c|c|}
\hline \multirow[b]{2}{*}{ Marker } & \multirow[b]{2}{*}{ Data Set } & \multirow[b]{2}{*}{ Total } & \multicolumn{4}{|c|}{ Cancer } & \multicolumn{4}{|c|}{ Normal } & \multirow[b]{2}{*}{$\begin{array}{c}\text { Total } \\
\text { Accuracy }\end{array}$} & \multirow[b]{2}{*}{ MCC } \\
\hline & & & $\begin{array}{c}\text { Cancer } \\
\text { Total }\end{array}$ & $\begin{array}{l}\text { Predict } \\
\text { Cancer }\end{array}$ & $\begin{array}{l}\text { Predict } \\
\text { Normal }\end{array}$ & Sensitivity & $\begin{array}{c}\text { Normal } \\
\text { Total }\end{array}$ & $\begin{array}{l}\text { Predict } \\
\text { Cancer }\end{array}$ & $\begin{array}{l}\text { Predict } \\
\text { Normal }\end{array}$ & Specificity & & \\
\hline \multirow{6}{*}{$\mathrm{CpG}$} & Training & 7003 & 4827 & 4734 & 93 & 0.981 & 2176 & 11 & 2165 & 0.995 & 0.985 & 0.966 \\
\hline & Validation & 571 & 370 & 352 & 18 & 0.951 & 201 & 10 & 191 & 0.95 & 0.951 & 0.894 \\
\hline & Test set 1 & 571 & 370 & 360 & 10 & 0.973 & 201 & 9 & 192 & 0.955 & 0.967 & 0.927 \\
\hline & Test set 2 & 3309 & 3041 & 2795 & 246 & 0.919 & 268 & 39 & 229 & 0.854 & 0.914 & 0.602 \\
\hline & Test set 3 & 2072 & 1532 & 1433 & 99 & 0.935 & 540 & 52 & 488 & 0.904 & 0.927 & 0.817 \\
\hline & All three test sets & 5952 & 4943 & 4588 & 355 & 0.928 & 1009 & 100 & 909 & 0.901 & 0.924 & 0.761 \\
\hline \multirow{6}{*}{ Promoter } & Training & 7003 & 4827 & 4676 & 151 & 0.969 & 2176 & 3 & 2173 & 0.999 & 0.978 & 0.951 \\
\hline & Validation & 571 & 370 & 354 & 16 & 0.957 & 201 & 5 & 196 & 0.975 & 0.963 & 0.921 \\
\hline & Test set 1 & 571 & 370 & 353 & 17 & 0.954 & 201 & 8 & 193 & 0.96 & 0.956 & 0.906 \\
\hline & Test set 2 & 3309 & 3041 & 2641 & 400 & 0.868 & 268 & 28 & 240 & 0.9 & 0.871 & 0.528 \\
\hline & Test set 3 & 2072 & 1532 & 1443 & 89 & 0.942 & 540 & 155 & 385 & 0.713 & 0.882 & 0.684 \\
\hline & All three test sets & 5952 & 4943 & 4437 & 506 & 0.898 & 1009 & 191 & 818 & 0.811 & 0.883 & 0.639 \\
\hline
\end{tabular}

Note: 'Predict cancer' or 'Predict normal' indicates samples predicted as cancer or normal. Training, Validation, Test set 1, Test set 2 and Test set 3 respectively indicate Training data set,

Validation data set, Test data set 1, Test data set 2 and Test data set 3. MCC indicates Matthews Correlation Coefficient [25]. 
Table 4. The prediction results of three data sets for $12 \mathrm{CpG}$ markers.

\begin{tabular}{|c|c|c|c|c|c|c|c|c|c|c|c|c|}
\hline \multirow[b]{2}{*}{ Data Set } & \multirow[b]{2}{*}{$\begin{array}{l}\text { Tissue } \\
\text { Types }\end{array}$} & \multirow[b]{2}{*}{ Total } & \multicolumn{4}{|c|}{ Cancer } & \multicolumn{4}{|c|}{ Normal } & \multirow[b]{2}{*}{$\begin{array}{c}\text { Total } \\
\text { Accuracy }\end{array}$} & \multirow[b]{2}{*}{ MCC } \\
\hline & & & $\begin{array}{c}\text { Cancer } \\
\text { Total }\end{array}$ & $\begin{array}{l}\text { Predict } \\
\text { Cancer }\end{array}$ & $\begin{array}{l}\text { Predict } \\
\text { Normal }\end{array}$ & Sensitivity & $\begin{array}{c}\text { Normal } \\
\text { Total }\end{array}$ & $\begin{array}{l}\text { Predict } \\
\text { Cancer }\end{array}$ & $\begin{array}{l}\text { Predict } \\
\text { Normal }\end{array}$ & Specificity & & \\
\hline \multirow{9}{*}{ Training } & Breast & 1122 & 1006 & 993 & 13 & 0.987 & 116 & 2 & 114 & 0.983 & 0.987 & 0.932 \\
\hline & Colorectal & 390 & 371 & 367 & 4 & 0.989 & 19 & 0 & 19 & 1 & 0.99 & 0.904 \\
\hline & Kidney & 794 & 593 & 573 & 20 & 0.966 & 201 & 0 & 201 & 1 & 0.975 & 0.937 \\
\hline & Leukocyte & 576 & 0 & 0 & 0 & - & 576 & 1 & 575 & 0.998 & 0.998 & 0 \\
\hline & Liver & 442 & 366 & 355 & 11 & 0.97 & 76 & 0 & 76 & 1 & 0.975 & 0.92 \\
\hline & Lung & 1155 & 857 & 839 & 18 & 0.979 & 298 & 2 & 296 & 0.993 & 0.983 & 0.956 \\
\hline & Prostate & 529 & 491 & 476 & 15 & 0.969 & 38 & 0 & 38 & 1 & 0.972 & 0.834 \\
\hline & Uterus & 432 & 416 & 415 & 1 & 0.998 & 16 & 0 & 16 & 1 & 0.998 & 0.969 \\
\hline & Breast & 85 & 60 & 60 & 0 & 1 & 25 & 1 & 24 & 0.96 & 0.988 & 0.972 \\
\hline \multirow{7}{*}{ Validation } & Colorectal & 56 & 40 & 40 & 0 & 1 & 16 & 2 & 14 & 0.875 & 0.964 & 0.913 \\
\hline & Kidney & 85 & 60 & 56 & 4 & 0.933 & 25 & 0 & 25 & 1 & 0.953 & 0.897 \\
\hline & Leukocyte & 40 & 0 & 0 & 0 & - & 40 & 0 & 40 & 1 & 1 & 0 \\
\hline & Liver & 60 & 40 & 40 & 0 & 1 & 20 & 0 & 20 & 1 & 1 & 1 \\
\hline & Lung & 120 & 80 & 73 & 7 & 0.912 & 40 & 1 & 39 & 0.975 & 0.933 & 0.86 \\
\hline & Prostate & 70 & 50 & 44 & 6 & 0.88 & 20 & 5 & 15 & 0.75 & 0.843 & 0.621 \\
\hline & Uterus & 55 & 40 & 39 & 1 & 0.975 & 15 & 1 & 14 & 0.933 & 0.964 & 0.908 \\
\hline \multirow{8}{*}{ Test 1} & Breast & 85 & 60 & 58 & 2 & 0.967 & 25 & 1 & 24 & 0.96 & 0.965 & 0.916 \\
\hline & Colorectal & 56 & 40 & 40 & 0 & 1 & 16 & 1 & 15 & 0.938 & 0.982 & 0.956 \\
\hline & Kidney & 85 & 60 & 58 & 2 & 0.967 & 25 & 0 & 25 & 1 & 0.976 & 0.946 \\
\hline & Leukocyte & 40 & 0 & 0 & 0 & - & 40 & 0 & 40 & 1 & 1 & 0 \\
\hline & Liver & 60 & 40 & 38 & 2 & 0.95 & 20 & 1 & 19 & 0.95 & 0.95 & 0.889 \\
\hline & Lung & 120 & 80 & 80 & 0 & 1 & 40 & 0 & 40 & 1 & 1 & 1 \\
\hline & Prostate & 70 & 50 & 46 & 4 & 0.92 & 20 & 5 & 15 & 0.75 & 0.871 & 0.681 \\
\hline & Uterus & 55 & 40 & 40 & 0 & 1 & 15 & 1 & 14 & 0.933 & 0.982 & 0.954 \\
\hline
\end{tabular}


Table 5. The prediction results of three data sets for 13 promoter markers.

\begin{tabular}{|c|c|c|c|c|c|c|c|c|c|c|c|c|}
\hline \multirow[b]{2}{*}{ Data Set } & \multirow{2}{*}{$\begin{array}{l}\text { Tissue } \\
\text { Types }\end{array}$} & \multirow[b]{2}{*}{ Total } & \multicolumn{4}{|c|}{ Cancer } & \multicolumn{4}{|c|}{ Normal } & \multirow[b]{2}{*}{$\begin{array}{c}\text { Total } \\
\text { Accuracy }\end{array}$} & \multirow[b]{2}{*}{$\mathrm{MCC}$} \\
\hline & & & $\begin{array}{c}\text { Cancer } \\
\text { Total }\end{array}$ & $\begin{array}{l}\text { Predict } \\
\text { Cancer }\end{array}$ & $\begin{array}{l}\text { Predict } \\
\text { Normal }\end{array}$ & Sensitivity & $\begin{array}{c}\text { Normal } \\
\text { Total }\end{array}$ & $\begin{array}{l}\text { Predict } \\
\text { Cancer }\end{array}$ & $\begin{array}{l}\text { Predict } \\
\text { Normal }\end{array}$ & Specificity & & \\
\hline \multirow{9}{*}{ Training } & Breast & 1122 & 1006 & 984 & 22 & 0.978 & 116 & 1 & 115 & 0.991 & 0.98 & 0.902 \\
\hline & Colorectal & 390 & 371 & 370 & 1 & 0.997 & 19 & 0 & 19 & 1 & 0.997 & 0.973 \\
\hline & Kidney & 794 & 593 & 545 & 48 & 0.919 & 201 & 0 & 201 & 1 & 0.94 & 0.861 \\
\hline & Leukocyte & 576 & 0 & 0 & 0 & - & 576 & 0 & 576 & 1 & 1 & 0 \\
\hline & Liver & 442 & 366 & 354 & 12 & 0.967 & 76 & 0 & 76 & 1 & 0.973 & 0.914 \\
\hline & Lung & 1155 & 857 & 829 & 28 & 0.967 & 298 & 0 & 298 & 1 & 0.976 & 0.94 \\
\hline & Prostate & 529 & 491 & 470 & 21 & 0.957 & 38 & 1 & 37 & 0.974 & 0.958 & 0.769 \\
\hline & Uterus & 432 & 416 & 416 & 0 & 1 & 16 & 1 & 15 & 0.938 & 0.998 & 0.967 \\
\hline & Breast & 85 & 60 & 59 & 1 & 0.983 & 25 & 0 & 25 & 1 & 0.988 & 0.972 \\
\hline \multirow{7}{*}{ Validation } & Colorectal & 56 & 40 & 40 & 0 & 1 & 16 & 0 & 16 & 1 & 1 & 1 \\
\hline & Kidney & 85 & 60 & 57 & 3 & 0.95 & 25 & 0 & 25 & 1 & 0.965 & 0.921 \\
\hline & Leukocyte & 40 & 0 & 0 & 0 & - & 40 & 0 & 40 & 1 & 1 & 0 \\
\hline & Liver & 60 & 40 & 40 & 0 & 1 & 20 & 0 & 20 & 1 & 1 & 1 \\
\hline & Lung & 120 & 80 & 70 & 10 & 0.875 & 40 & 0 & 40 & 1 & 0.917 & 0.837 \\
\hline & Prostate & 70 & 50 & 48 & 2 & 0.96 & 20 & 3 & 17 & 0.85 & 0.929 & 0.823 \\
\hline & Uterus & 55 & 40 & 40 & 0 & 1 & 15 & 2 & 13 & 0.867 & 0.964 & 0.909 \\
\hline \multirow{8}{*}{ Test set 1} & Breast & 85 & 60 & 57 & 3 & 0.95 & 25 & 0 & 25 & 1 & 0.965 & 0.921 \\
\hline & Colorectal & 56 & 40 & 40 & 0 & 1 & 16 & 0 & 16 & 1 & 1 & 1 \\
\hline & Kidney & 85 & 60 & 56 & 4 & 0.933 & 25 & 0 & 25 & 1 & 0.953 & 0.897 \\
\hline & Leukocyte & 40 & 0 & 0 & 0 & - & 40 & 0 & 40 & 1 & 1 & 0 \\
\hline & Liver & 60 & 40 & 38 & 2 & 0.95 & 20 & 1 & 19 & 0.95 & 0.95 & 0.889 \\
\hline & Lung & 120 & 80 & 77 & 3 & 0.963 & 40 & 0 & 40 & 1 & 0.975 & 0.946 \\
\hline & Prostate & 70 & 50 & 45 & 5 & 0.9 & 20 & 5 & 15 & 0.75 & 0.857 & 0.65 \\
\hline & Uterus & 55 & 40 & 40 & 0 & 1 & 15 & 2 & 13 & 0.867 & 0.964 & 0.909 \\
\hline
\end{tabular}


Table 6. The prediction results of two test data sets for $12 \mathrm{CpG}$ markers.

\begin{tabular}{|c|c|c|c|c|c|c|c|c|c|c|c|c|}
\hline \multirow[b]{2}{*}{ Data Set } & \multirow{2}{*}{ Tissue Types } & \multirow[b]{2}{*}{ Total } & \multicolumn{4}{|c|}{ Cancer } & \multicolumn{4}{|c|}{ Normal } & \multirow[b]{2}{*}{$\begin{array}{c}\text { Total } \\
\text { Accuracy }\end{array}$} & \multirow[b]{2}{*}{ MCC } \\
\hline & & & $\begin{array}{c}\text { Cancer } \\
\text { Total }\end{array}$ & $\begin{array}{l}\text { Predict } \\
\text { Cancer }\end{array}$ & $\begin{array}{l}\text { Predict } \\
\text { Normal }\end{array}$ & Sensitivity & $\begin{array}{c}\text { Normal } \\
\text { Total }\end{array}$ & $\begin{array}{l}\text { Predict } \\
\text { Cancer }\end{array}$ & $\begin{array}{l}\text { Predict } \\
\text { Normal }\end{array}$ & Specificity & & \\
\hline \multirow{15}{*}{$\begin{array}{c}\text { Test data } \\
\text { set } 2\end{array}$} & Adrenal gland & 267 & 264 & 213 & 51 & 0.807 & 3 & 0 & 3 & 1 & 0.809 & 0.212 \\
\hline & Bile duct & 45 & 36 & 36 & 0 & 1 & 9 & 0 & 9 & 1 & 1 & 1 \\
\hline & Bladder & 440 & 419 & 411 & 8 & 0.981 & 21 & 3 & 18 & 0.857 & 0.975 & 0.758 \\
\hline & Esophagus & 202 & 186 & 185 & 1 & 0.995 & 16 & 5 & 11 & 0.688 & 0.97 & 0.779 \\
\hline & Eyes & 80 & 80 & 74 & 6 & 0.925 & 0 & 0 & 0 & - & 0.925 & 0 \\
\hline & Head and neck & 580 & 530 & 529 & 1 & 0.998 & 50 & 10 & 40 & 0.8 & 0.981 & 0.874 \\
\hline & Lymph nodes & 51 & 48 & 46 & 2 & 0.958 & 3 & 0 & 3 & 1 & 0.961 & 0.758 \\
\hline & Oral & 104 & 65 & 46 & 19 & 0.708 & 39 & 2 & 37 & 0.949 & 0.798 & 0.637 \\
\hline & Ovary & 10 & 10 & 10 & 0 & 1 & 0 & 0 & 0 & - & 1 & 0 \\
\hline & Pancreas & 391 & 352 & 265 & 87 & 0.753 & 39 & 3 & 36 & 0.923 & 0.77 & 0.436 \\
\hline & Pleura & 87 & 87 & 81 & 6 & 0.931 & 0 & 0 & 0 & - & 0.931 & 0 \\
\hline & Small bowel & 56 & 28 & 27 & 1 & 0.964 & 28 & 4 & 24 & 0.857 & 0.911 & 0.826 \\
\hline & Soft tissue & 269 & 265 & 250 & 15 & 0.943 & 4 & 0 & 4 & 1 & 0.944 & 0.446 \\
\hline & Testis & 156 & 156 & 135 & 21 & 0.865 & 0 & 0 & 0 & - & 0.865 & 0 \\
\hline & Thyroid & 571 & 515 & 487 & 28 & 0.946 & 56 & 12 & 44 & 0.786 & 0.93 & 0.655 \\
\hline \multirow{5}{*}{$\begin{array}{c}\text { Test data } \\
\text { set } 3\end{array}$} & Bone marrow & 386 & 325 & 257 & 68 & 0.791 & 61 & 0 & 61 & 1 & 0.824 & 0.611 \\
\hline & Cervix & 356 & 315 & 311 & 4 & 0.987 & 41 & 1 & 40 & 0.976 & 0.986 & 0.934 \\
\hline & Nasopharynx & 48 & 24 & 19 & 5 & 0.792 & 24 & 2 & 22 & 0.917 & 0.854 & 0.714 \\
\hline & Skin & 694 & 473 & 466 & 7 & 0.985 & 221 & 1 & 220 & 0.995 & 0.988 & 0.974 \\
\hline & Stomach & 588 & 395 & 380 & 15 & 0.962 & 193 & 48 & 145 & 0.751 & 0.893 & 0.753 \\
\hline
\end{tabular}


Table 7. The prediction results of two test data sets for 13 promoter markers.

\begin{tabular}{|c|c|c|c|c|c|c|c|c|c|c|c|c|}
\hline \multirow[b]{2}{*}{ Data Set } & \multirow{2}{*}{ Tissue Types } & \multirow[b]{2}{*}{ Total } & \multicolumn{4}{|c|}{ Cancer } & \multicolumn{4}{|c|}{ Normal } & \multirow[b]{2}{*}{$\begin{array}{c}\text { Total } \\
\text { Accuracy }\end{array}$} & \multirow[b]{2}{*}{ MCC } \\
\hline & & & $\begin{array}{c}\text { Cancer } \\
\text { Total }\end{array}$ & $\begin{array}{l}\text { Predict } \\
\text { Cancer }\end{array}$ & $\begin{array}{c}\text { Predict } \\
\text { Normal }\end{array}$ & Sensitivity & $\begin{array}{c}\text { Normal } \\
\text { Total }\end{array}$ & $\begin{array}{l}\text { Predict } \\
\text { Cancer }\end{array}$ & $\begin{array}{l}\text { Predict } \\
\text { Normal }\end{array}$ & Specificity & & \\
\hline \multirow{15}{*}{$\begin{array}{c}\text { Test data } \\
\text { set } 2\end{array}$} & Adrenal gland & 267 & 264 & 251 & 13 & 0.951 & 3 & 0 & 3 & 1 & 0.951 & 0.422 \\
\hline & Bile duct & 45 & 36 & 36 & 0 & 1 & 9 & 0 & 9 & 1 & 1 & 1 \\
\hline & Bladder & 440 & 419 & 414 & 5 & 0.988 & 21 & 3 & 18 & 0.857 & 0.982 & 0.81 \\
\hline & Esophagus & 202 & 186 & 186 & 0 & 1 & 16 & 7 & 9 & 0.562 & 0.965 & 0.736 \\
\hline & Eyes & 80 & 80 & 74 & 6 & 0.925 & 0 & 0 & 0 & - & 0.925 & 0 \\
\hline & Head and neck & 580 & 530 & 523 & 7 & 0.987 & 50 & 6 & 44 & 0.88 & 0.978 & 0.859 \\
\hline & Lymph nodes & 51 & 48 & 48 & 0 & 1 & 3 & 3 & 0 & 0 & 0.941 & 0 \\
\hline & Oral & 104 & 65 & 37 & 28 & 0.569 & 39 & 2 & 37 & 0.949 & 0.712 & 0.518 \\
\hline & Ovary & 10 & 10 & 10 & 0 & 1 & 0 & 0 & 0 & - & 1 & 0 \\
\hline & Pancreas & 391 & 352 & 297 & 55 & 0.844 & 39 & 3 & 36 & 0.923 & 0.852 & 0.544 \\
\hline & Pleura & 87 & 87 & 82 & 5 & 0.943 & 0 & 0 & 0 & - & 0.943 & 0 \\
\hline & Small bowel & 56 & 28 & 27 & 1 & 0.964 & 28 & 2 & 26 & 0.929 & 0.946 & 0.893 \\
\hline & Soft tissue & 269 & 265 & 263 & 2 & 0.992 & 4 & 0 & 4 & 1 & 0.993 & 0.813 \\
\hline & Testis & 156 & 156 & 134 & 22 & 0.859 & 0 & 0 & 0 & - & 0.859 & 0 \\
\hline & Thyroid & 571 & 515 & 259 & 256 & 0.503 & 56 & 2 & 54 & 0.964 & 0.548 & 0.279 \\
\hline \multirow{5}{*}{$\begin{array}{c}\text { Test data } \\
\text { set } 3\end{array}$} & Bone marrow & 386 & 325 & 261 & 64 & 0.803 & 61 & 0 & 61 & 1 & 0.834 & 0.626 \\
\hline & Cervix & 356 & 315 & 310 & 5 & 0.984 & 41 & 0 & 41 & 1 & 0.986 & 0.937 \\
\hline & Nasopharynx & 48 & 24 & 9 & 15 & 0.375 & 24 & 0 & 24 & 1 & 0.688 & 0.48 \\
\hline & Skin & 694 & 473 & 470 & 3 & 0.994 & 221 & 1 & 220 & 0.995 & 0.994 & 0.987 \\
\hline & Stomach & 588 & 395 & 393 & 2 & 0.995 & 193 & 154 & 39 & 0.202 & 0.735 & 0.363 \\
\hline
\end{tabular}




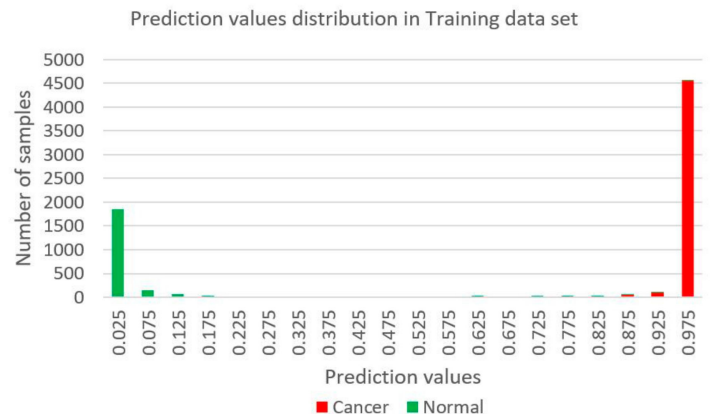

(a)

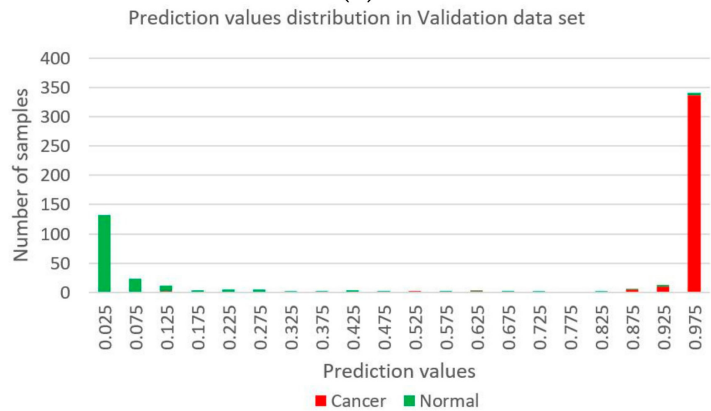

(c)

Prediction values distribution in all three test data set

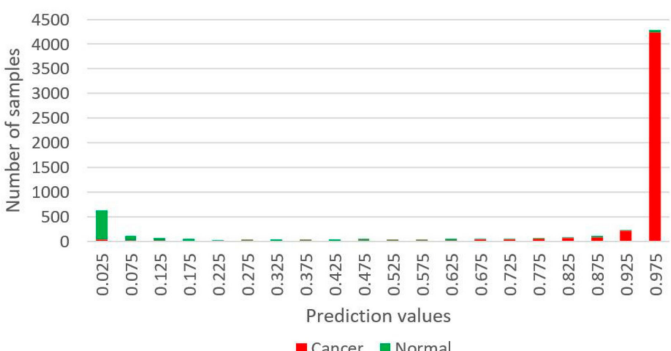

(e)

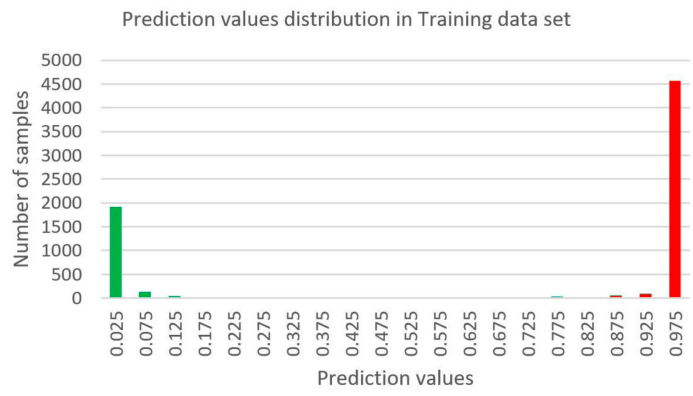

- Cancer a Normal

(b)

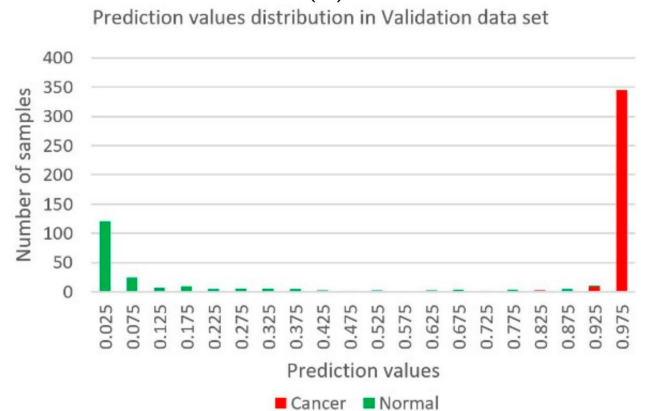

(d)

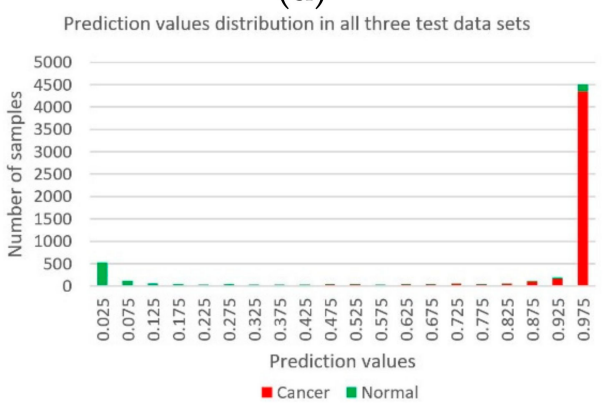

(f)

Figure 5. The distribution of prediction values in all samples. (a,c,e) come from $12 \mathrm{CpG}$ markers and $(\mathbf{b}, \mathbf{d}, \mathbf{f})$ come from 13 promoter markers. Red indicates the status of the sample is cancer, and green indicates the status of the sample is normal.

Interpreting model predictions becomes more and more crucial in the field of machine learning, especially for deep learning. An outstanding approach has been proposed, which used SHAP (SHapley Additive exPlanation) values as a unified measure of feature importance [26]. Figure 6 shows the average absolute SHAP value of each marker. For CpG markers, cg07333191 has biggest impact on model output, while cg04374393 has least impact. For promoter markers, AURKB has biggest impact on model output, while the impact of ACVRL1 is least. Figure S4 shows the detailed impact of each marker to the model output in four samples. 


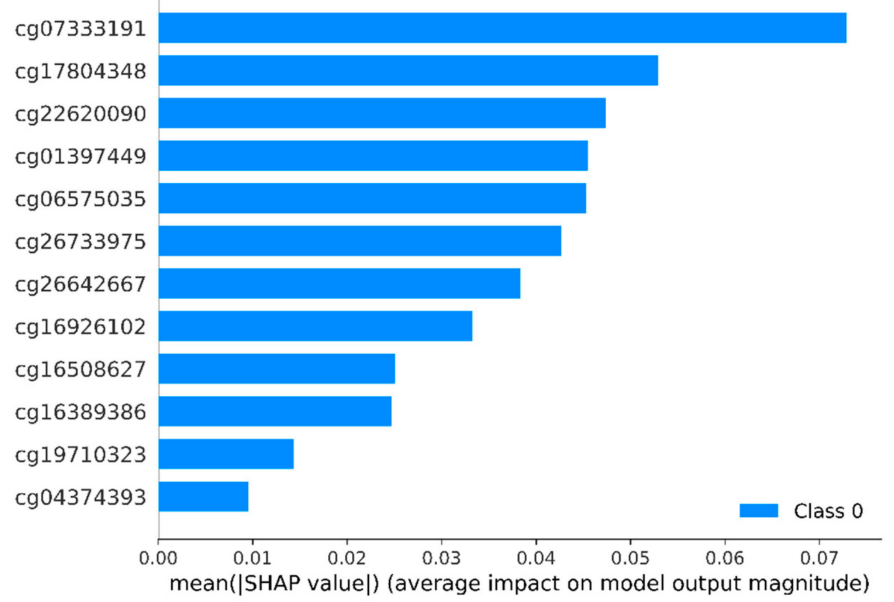

(a)

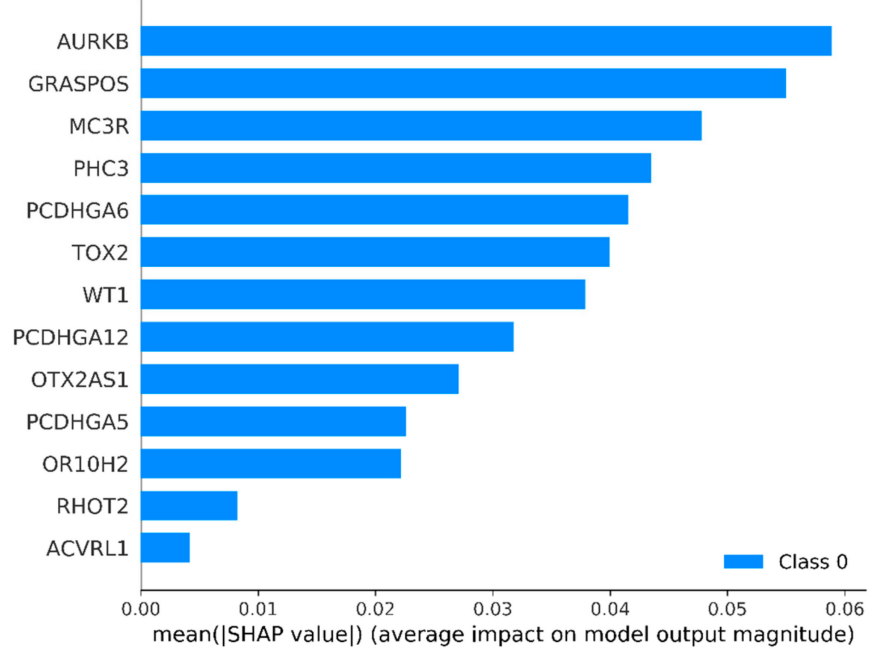

(b)

Figure 6. The distribution of average absolute SHAP value for all markers. (a) comes from $12 \mathrm{CpG}$ markers and (b) comes from 13 promoter markers. SHAP: SHapley Additive explanation.

\subsection{Evaluating Reliability of Markers and Diagnostic Prediction Models}

To verify whether our deep learning models perform better than general traditional machine learning strategy, such as logistic regression, we fitted our data in two logistic regression models. The results indicate that deep learning predicting method performs more precise than logistic regression method in our data sets actually (Figure S2, Table S8). To test the reliability of the selected markers, we randomly partitioned all samples into $80 \%$ for training, $10 \%$ for validation, and $10 \%$ for testing. We constructed two other deep learning models for CpG markers and promoter markers, and fed all these samples into the models. Figure S3 shows ROC of the three data sets, and AUCs (0.993 for CpG markers and 0.995 for promoter markers) demonstrate that the selected markers can classify all samples precisely. The robustness of biomarkers for cancer diagnosis or prognosis might be low due to tumor heterogeneity, and random sampling was suggested to evaluate the robustness of markers [27]. We performed random sampling of 100 times, data were divided into one training data set and one test data set each time. Training data set was used to train models and test data set was used to evaluate the performance of models. Figure S5 shows predict accuracies for both CpG markers and promoter markers. The result indicates the robustness of our markers is strong, since the predict accuracies are high in different data sets. To test performance of the markers in liquid biopsy, we utilized the markers to predict cell-free DNA methylation data of 163 prostate cancer samples. Sensitivity for CpG markers 
was $100 \%$, and for promoter markers was $92 \%$. Additionally, another dataset whose GEO accession number is GSE110185 contains six cell-free DNA pooled samples (two colorectal cancer, two advanced adenomas and two healthy control samples). Additionally, all of these six samples were predicted as normal samples. Notably, for both marker types, specificity of normal whole blood is $100 \%$, and whole blood samples are the most similar samples to cell-free DNA samples.

\section{Discussion}

Most related studies identifying methylation markers focused on one or a few cancer types. The most important impact for our study is that we attempted to identify two categories of methylation markers, CpG markers and promoter markers, to classify and predict pan-cancers. The reliability of this study lies in the fact that all three test data sets were tested only once to avoid overfitting. Therefore, the predict results we show here can prove that pan-cancers can be predicted precisely by the selected methylation markers. Sensitivity and specificity in most cancer types are high enough for both markers. Nonetheless, for promoter markers, specificities of two cancer types (esophagus and stomach cancer) and sensitivities of three cancer types (oral, thyroid, and nasopharynx cancer) are too low to predict precisely. Sensitivity and specificity of these five cancer types are high enough for CpG markers, which means the samples are qualified. Therefore, a possible reason is that in these samples, $\mathrm{CPG}$ probes located at promoters are not enough to calculate promoter methylation values precisely. This is the potential defect of promoter markers that promoter methylation value calculating might be inaccurate since each promoter has different length definition actually. Another possible reason is that $\mathrm{CpG}$ markers may be more adapted to these cancer types than promoter markers. Nonetheless, identifying promoter methylation markers is worth attempting, since promoter has a close relation with the process of cancer developing. The advantage of pan-cancer methylation biomarkers is that diagnosis of diverse cancer types can be based on targeted measuring of these biomarkers. Therefore, these biomarkers could be applied in liquid biopsy effectively. The performance of the selected markers in cell-free DNA methylation data of 163 cancer samples was excellent. However, for GSE110185 dataset, all six pooled samples were predicted as normal samples. Two advanced adenomas samples should be regarded as non-cancer samples, thus the prediction accuracy is 0.667. However, because lack of abundant normal cell-free DNA samples, specificity remains to be verified in more normal samples. We have put arguments of the well-trained deep learning models online to let more researchers validate the reliability of our model. What should be emphasized is the dependability of cell-free DNA samples. Since in the process of cell-free DNA isolation, contamination could easily happen, such as ruptured blood cells [28]. Therefore, samples containing cell-free DNA are prone to be classified as normal samples.

Comparing other studies to our study, Vrba et al. [15] attempted to identify CpG markers to predict pan-cancer. One difference between their strategy and ours is that they reduced the number of markers by comparing cancer samples to mix unrelated normal whole blood samples. While we identified markers by comparing cancer samples to mix matched normal samples. Another difference is that they identified markers in each cancer, and summarized all markers to one marker set. However, our strategy involves gathering all samples from the start, and identifying markers fitting all data. Due to a lack of cell-free DNA methylation data, one compromise in their research is that they treated whole blood samples as cell-free DNA samples simulation. Although whole blood samples mainly contained leukocytes, whole blood samples are the most similar samples to cell-free samples. Therefore, specificity for cell-free DNA samples in our study could be calculated by whole blood approximately, which means for both two marker types, specificity of whole blood is $100 \%$. In our study, taking the intersection of markers from two machine learning strategies to reduce the number of markers is a compromising strategy. In the future, more convincing statistics, machine learning, and data dividing strategies for mining marker are necessary. With a lack of abundant cell-free DNA samples, more verification results depend on more researchers using our models published online to classify cell-free DNA samples. Additionally, the pipeline of this study can be applied in cell-free DNA samples to identify methylation markers more adaptive to cell-free DNA samples. The long-range perspective is 
identifying one methylation markers set for cell-free DNA samples, applying them to cancer early diagnosis for pan-cancers, and making all cancers be exposed early, be cured early, to reduce death rate of cancers. The models we have trained can only diagnose whether a sample is cancer or normal tissue, but cannot judge which cancer type the sample belongs to. Multiple classification models need to be constructed to diagnose the exact cancer type of samples in future study.

\section{Conclusions}

In our study, we collected whole genome methylation data of 10,140 cancer samples and 3386 normal samples, and divided them into five data sets. Using three machine learning methods, we identified two categories of markers: $12 \mathrm{CpG}$ markers and 13 promoter markers. Three of $12 \mathrm{CpG}$ markers and four of 13 promoter markers located at cancer-related genes. The performances of these markers in solid or cell-free DNA samples are both pretty good. Additionally, if the prediction result of one sample is the same between CpG markers and promoter markers, the prediction will be more reliable. To conclude, we found it possible to identify methylation markers used to predict pan-cancer. The long-range perspective is identifying one methylation markers set for efficient and precise liquid biopsy of pan-cancers.

Supplementary Materials: The following are available online at http:/www.mdpi.com/2073-4425/10/10/778/s1, Figure S1: The cost and accuracy change in training progress, Figure S2: ROC of logistic regression for the three data sets, Figure S3: ROC of the randomly partition three data set, Figure S4: The detailed impact of each marker to the model output in four samples, Figure S5: Plot of accuracy with random sampling, Table S1: The overview of Training data set, Validation data set, and Test data set 1, Table S2: The sample details of 4840 cancer samples and 1742 matched normal samples (All were divided into Training data set, Validation data set and Test data set 1 as Table S1 shows), Table S3: The sample details of 727 cancer samples and 836 normal samples (All were added into Training data set), Table S4: The sample details of 3041 cancer samples and 268 matched normal samples (All were added into Test data set 2), Table S5: The sample details of 1532 cancer samples and 540 virtual normal samples (All were added into Test data set 3), Table S6: The sample details of cell-free DNA samples, Table S7: The range of hyper-parameters during random searching, Table S8: The prediction accuracy contrast between Deep learning and Logistic regression.

Author Contributions: S.C.L. and S.Y. supervised the research and revised the manuscripts; B.L. collected the data, performed analysis and wrote the manuscript; Y.L. participated in the literature review and performed some analysis; X.P. and M.L. participated in the literature review; All authors discussed the results and reviewed the manuscript.

Funding: This research received no external funding.

Acknowledgments: Computing resource was supported by BGI-Shenzhen. The results here are in whole based on data generated by the TCGA Research Network: http://cancergenome.nih.gov/, and NCBI GEO Network: https://www.ncbi.nlm.nih.gov/geo/.

Conflicts of Interest: The authors declare no conflicts of interest.

\section{References}

1. Bird, A. Perceptions of epigenetics. Nature 2007, 447, 396-398. [CrossRef] [PubMed]

2. Vaissière, T.; Sawan, C.; Herceg, Z. Epigenetic interplay between histone modifications and DNA methylation in gene silencing. Mutat. Res./Rev. Mutat. Res. 2008, 659, 40-48.

3. Suzuki, M.M.; Bird, A. DNA methylation landscapes: Provocative insights from epigenomics. Nat. Rev. Genet. 2008, 9, 465-476. [CrossRef] [PubMed]

4. Bird, A.P.; Wolffe, A.P. Methylation-induced repression-Belts, braces, and chromatin. Cell 1999, 99, 451-454. [CrossRef]

5. Herman, J.G.; Baylin, S.B. Gene silencing in cancer in association with promoter hypermethylation. N. Engl. J. Med. 2003, 349, 2042-2054. [CrossRef] [PubMed]

6. Baylin, S.B. DNA methylation and gene silencing in cancer. Nat. Rev. Clin. Oncol. 2005, 2, S4-S11. [CrossRef]

7. Dong, Y.; Zhao, H.; Li, H.; Li, X.; Yang, S. DNA methylation as an early diagnostic marker of cancer. Biomed. Rep. 2014, 2, 326-330. [CrossRef]

8. Chen, Y.; Li, J.; Yu, X.; Li, S.; Zhang, X.; Mo, Z.; Hu, Y. APC gene hypermethylation and prostate cancer: A systematic review and meta-analysis. Eur. J. Hum. Genet. 2013, 21, 929-935. [CrossRef] 
9. Rivera, A.L.; Pelloski, C.E.; Gilbert, M.R.; Colman, H.; De La Cruz, C.; Sulman, E.P.; Bekele, B.N.; Aldape, K.D. MGMT promoter methylation is predictive of response to radiotherapy and prognostic in the absence of adjuvant alkylating chemotherapy for glioblastoma. Neuro-Oncology 2009, 12, 116-121. [CrossRef]

10. Xu, R.-H.; Wei, W.; Krawczyk, M.; Wang, W.; Luo, H.; Flagg, K.; Yi, S.; Shi, W.; Quan, Q.; Li, K. Circulating tumour DNA methylation markers for diagnosis and prognosis of hepatocellular carcinoma. Nat. Mater. 2017, 16, 1155-1161. [CrossRef]

11. Mikeska, T.; Craig, J. DNA methylation biomarkers: Cancer and beyond. Genes 2014, 5, 821-864. [CrossRef] [PubMed]

12. Weinstein, J.N.; Collisson, E.A.; Mills, G.B.; Shaw, K.R.M.; Ozenberger, B.A.; Ellrott, K.; Shmulevich, I.; Sander, C.; Stuart, J.M.; Cancer Genome Atlas Research Network. The cancer genome atlas pan-cancer analysis project. Nat. Genet. 2013, 45, 1113-1120. [CrossRef] [PubMed]

13. Witte, T.; Plass, C.; Gerhauser, C. Pan-cancer patterns of DNA methylation. Genome Med. 2014, 6, 66. [CrossRef] [PubMed]

14. Yang, X.; Gao, L.; Zhang, S. Comparative pan-cancer DNA methylation analysis reveals cancer common and specific patterns. Brief. Bioinform. 2016, 18, 761-773. [CrossRef]

15. Vrba, L.; Futscher, B.W. A suite of DNA methylation markers that can detect most common human cancers. Epigenetics 2018, 13, 61-72. [CrossRef] [PubMed]

16. Barrett, T.; Wilhite, S.E.; Ledoux, P.; Evangelista, C.; Kim, I.F.; Tomashevsky, M.; Marshall, K.A.; Phillippy, K.H.; Sherman, P.M.; Holko, M. NCBI GEO: Archive for functional genomics data sets-update. Nucleic Acids Res. 2012, 41, D991-D995. [CrossRef]

17. Huang, W.-Y.; Hsu, S.-D.; Huang, H.-Y.; Sun, Y.-M.; Chou, C.-H.; Weng, S.-L.; Huang, H.-D. MethHC: A database of DNA methylation and gene expression in human cancer. Nucleic Acids Res. 2014, 43, D856-D861. [CrossRef] [PubMed]

18. Pan, X.; Liu, B.; Wen, X.; Liu, Y.; Zhang, X.; Li, S. D-GPM: A deep learning method for gene promoter methylation inference. bioRxiv 2018. [CrossRef]

19. Gentleman, R.; Carey, V.; Huber, W.; Irizarry, R.; Dudoit, S. Bioinformatics and Computational Biology Solutions Using $R$ and Bioconductor; Springer Science \& Business Media: New York, NY, USA, 2006.

20. Benjamini, Y.; Hochberg, Y. Controlling the false discovery rate: A practical and powerful approach to multiple testing. J. R. Stat. Soc. Ser. B 1995, 57, 289-300. [CrossRef]

21. Tibshirani, R. Regression shrinkage and selection via the lasso. J. R. Stat. Soc. Ser. B 1996, 58, $267-288$. [CrossRef]

22. Diaz-Uriarte, R. GeneSrF and varSelRF: A web-based tool and R package for gene selection and classification using random forest. BMC Bioinform. 2007, 8, 328. [CrossRef] [PubMed]

23. Bergstra, J.; Bengio, Y. Random search for hyper-parameter optimization. J. Mach. Learn. Res. 2012, 13, 281-305.

24. Abadi, M.; Barham, P.; Chen, J.; Chen, Z.; Davis, A.; Dean, J.; Devin, M.; Ghemawat, S.; Irving, G.; Isard, M. Tensorflow: A system for large-scale machine learning. In Proceedings of the 12th USENIX Symposium on Operating Systems Design and Implementation (OSDI '16), Savannah, GA, USA, 2-4 November 2016; pp. 265-283.

25. Matthews, B.W. Comparison of the predicted and observed secondary structure of T4 phage lysozyme. Biochim. Biophys. Acta (Bba)-Protein Struct. 1975, 405, 442-451. [CrossRef]

26. Lundberg, S.M.; Lee, S.-I. A unified approach to interpreting model predictions. In Proceedings of the 31st Conference on Neural Information Processing Systems (NIPS 2017), Long Beach, CA, USA, 4-9 December 2017; pp. 4765-4774.

27. Li, J.; Lenferink, A.E.; Deng, Y.; Collins, C.; Cui, Q.; Purisima, E.O.; O'Connor-McCourt, M.D.; Wang, E. Identification of high-quality cancer prognostic markers and metastasis network modules. Nat. Commun. 2010, 1, 34. [CrossRef] [PubMed]

28. Lui, Y.Y.; Chik, K.-W.; Chiu, R.W.; Ho, C.-Y.; Lam, C.W.; Lo, Y.D. Predominant hematopoietic origin of cell-free DNA in plasma and serum after sex-mismatched bone marrow transplantation. Clin. Chem. 2002, 48, 421-427. [PubMed] 